\title{
Amor, trabajo y (des) protección social.
}

\section{Una aproximación al cuidado no remunerado de personas mayores en la ciudad de Mar del Plata, Argentina}

Love, work and (no) social protection. An approach to the unpaid care of the elderly in the city of Mar del Plata, Argentina

Romina Cutuli

Doctora en Historia

(Universidad Nacional de Mar del Plata)

Investigadora Adjunta (CONICET)

Integrante del Grupo de Estudios del Trabajo,

Facultad de Ciencias Económicas y Sociales

(Universidad Nacional de Mar del Plata)

Correo: rominacutuli@yahoo.com.ar

Rosario Camou

Licenciada en Trabajo Social

(Universidad Nacional de Mar del Plata)

Correo: rochi_camou@hotmail.com
Antonella Colombo

Licenciada en Trabajo Social

(Universidad Nacional de Mar del Plata)

Correo: colomboantonella@outlook.com

Marianella Vinitzky

Licenciada en Trabajo Social

(Universidad Nacional de Mar del Plata)

Correo: marianelavinitzky@hotmail.com 


\section{Resumen}

En este artículo se analizan las condiciones de familiarización y feminización del cuidado de personas mayores en la ciudad de Mar del Plata, Argentina. En el país, la organización social del cuidado y las normativas que la regulan están signadas por un marcado familiarismo, lo que restringe las oportunidades de autonomía de las personas cuidadoras y limita el acceso a cuidados de calidad y bienestar material a las personas mayores. El Estado se hace presente en situaciones de extrema gravedad o ausencia de redes familiares, imponiendo la tensión entre el abandono y la resignación del proyecto de vida propio. La investigación que se recupera en este artículo se inició en un contexto anterior a la declaración de la pandemia y continuó, con adaptaciones metodológicas, en los primeros dos meses de pandemia. Recuperamos, a través de entrevistas y un cuestionario autoadministrado, la experiencia de mujeres cuidadoras no remuneradas de personas mayores, su perspectiva sobre el impacto del cuidado no remunerado en sus proyectos de vida y las representaciones subjetivas en torno a la labor que realizan.

\section{Palabras clave}

Trabajo, Género, Organización del cuidado, Cuidado no remunerado, Personas mayores.

\section{Abstract}

This article analyzes the conditions of familiarization and feminization of elderly care in the city of Mar del Plata, Argentina. In the country, the social organization of care and the regulations that regulate it are marked by a marked familiarity, which restricts the opportunities for autonomy of caregivers and limits access to quality care and material well-being for the elderly. The State is present in situations of extreme gravity or absence of family networks, imposing the tension between abandonment and resignation of one's own life project. The research recovered in this article began in a context prior to the declaration of the pandemic and continued, with methodological adaptations, in the first two months of the pandemic. We recover, through interviews and a self-administered questionnaire, the experience of unpaid caregivers of the elderly, their perspective on the impact of unpaid care on their life projects and subjective representations about the work they do.

\section{Keywords}

Work, Gender, Care organization, Unpaid care, Elder people. 


\section{Introducción}

Sin embargo, Tita no estaba conforme. Una gran cantidad de dudas e inquietudes acudían a su mente. Por ejemplo, le agradaría tener conocimiento de quién había iniciado esta tradición familiar. Sería bueno hacerle saber a esta ingeniosa persona que en su perfecto plan para asegurar la vejez de las mujeres había una ligera falla. Si Tita no podía casarse ni tener hijos, ¿quién la cuidaría entonces al llegar a la senectud? ¿Cuál era la solución acertada en estos casos? ¿O es que no se esperaba que las hijas que se quedaban a cuidar a sus madres sobrevivieran mucho tiempo después del fallecimiento de sus progenitoras? ¿Y dónde se quedaban las mujeres que se casaban y no podían tener hijos, quién se encargaría de atenderlas? Es más, quería saber, ¿cuáles fueron las investigaciones que se llevaron a cabo para concluir que la hija menor era la más indicada para velar por su madre y no la hija mayor? ¿Se había tomado alguna vez en cuenta la opinión de las hijas afectadas? ¿Le estaba permitido, al menos, si es que no se podía casar, conocer el amor? ¿O ni siquiera eso?

Laura Esquivel, Como agua para chocolate, 1989

Al igual que a Tita, generación tras generación, a las mujeres les fue impuesto el cuidado a costa de sus propios proyectos de vida y sin permitirles siquiera pensar en otra posibilidad. Reproduciendo tradiciones y, con ello, privilegios, el trabajo no remunerado realizado por mujeres sigue ocupando un rol central en la provisión social de bienestar a las personas mayores. Pese a las múltiples transformaciones culturales y sociodemográficas a las que asistimos en las últimas décadas, miles de Titas entregan su vida al cuidado sin que nadie responda a esas preguntas que es más cómodo no hacer. En este artículo se abordarán, como un fenómeno persistente, las desigualdades de género en la organización social del cuidado de personas mayores en la ciudad de Mar del Plata. En particular, la centralidad del trabajo no remunerado desempeñado mayoritariamente por mujeres en la provisión social de bienestar. Se propone un enfoque interseccional, entendiéndolo como la forma particular en la que diferentes categorías de opresión -como la raza, el género, la sexualidad y la nacionalidad- se encuentran y se corporizan en las personas (Collins, 2000).

La ciudad de Mar del Plata, donde se ha desarrollado este estudio, tiene una estructura poblacional envejecida, como consecuencia no solo de la permanencia sino también de la migración de personas ma- 
yores, en especial del área metropolitana de Buenos Aires (en adelante, AMBA) en busca de una calidad de vida adecuada para esta etapa del ciclo vital. Según la Convención establecida por Naciones Unidas (Naciones Unidas, 2015), una población es considerada envejecida cuando tiene más del 7\% de personas mayores de 60 años. Mar del Plata es considerada como una de las ciudades con mayor índice de envejecimiento poblacional del país, con el $16 \%$ de habitantes mayores de 65 años de edad (INDEC-Censo 2010).

El cuidado de personas mayores, al igual que el conjunto de las tareas vinculadas con la sostenibilidad de la vida (Carrasco, 2001) está atravesado por un escenario de revolución estancada (Hochshild, 1989; Wainerman, 2005). Es decir, una división sexual del trabajo al interior de los hogares que no se modificó al ritmo de la mayor participación de las mujeres en el trabajo remunerado. Tomaremos como estudio de caso la ciudad de Mar del Plata, con especificidades vinculadas a una organización del cuidado familiarizada y necesidades superiores a la media nacional, en virtud del envejecimiento poblacional. A partir de experiencias de personas que ejercen cuidado no remunerado a personas mayores -casi todas ellas mujeres- analizaremos los condicionantes que llevan a ser las principales responsables del cuidado, así como las limitaciones al despliegue de un proyecto de vida propio en virtud de la sobrecarga de trabajo de cuidado. Finalmente haremos algunas reflexiones que emergen de esta investigación para contribuir a la construcción de una agenda de políticas de cuidado promotora del bienestar de dependientes y cuidadores.

\section{Metodología}

El análisis se desarrolla a partir de un estudio de caso. Como se ha mencionado antes, las particularidades demográficas de la ciudad hacen de ella un "laboratorio" de particular relevancia por su envejecimiento poblacional, tendencia en la que la ciudad se encontraría a la vanguardia en comparación con la media nacional. La población de 65 años y más representa el 16\% Mar del Plata y el 10\% Argentina, según los datos del último censo nacional (INDEC, 2010). El porcentaje de personas mayores en esta ciudad equivale al proyectado a 
nivel mundial por las Naciones Unidas para el 2050 (UN, 2021). Esta condición permitiría proyectar, a partir de los desafíos actuales de la organización social del cuidado en la ciudad, políticas públicas de cuidado de personas mayores con perspectiva de género e interseccional.

La planificación inicial de la investigación se vio afectada por el aislamiento social y preventivo obligatorio (ASPO) dictaminado por el gobierno nacional en el mes de marzo de 2020, como medida de emergencia frente a la pandemia COVID-19¹ . Para entonces, se había iniciado un abordaje cualitativo a través de entrevistas semiestructuradas presenciales, técnica que debió revisarse en virtud de la emergencia sanitaria. Así, entre los meses de abril y mayo, se decidió incorporar otro mecanismo de acercamiento a las personas que ejercen cuidado no remunerado. En ese marco, se diseñó un cuestionario digital autoadministrado (Hernández Sampieri, 2014) a través de la plataforma Google Forms, con 39 preguntas cerradas y abiertas. Su distribución se realizó a través de redes sociales, con una breve nota explicativa sobre los objetivos de la investigación, las referencias institucionales y el compromiso de anonimización de los datos. Además, se facilitó un e-mail de contacto para ampliar información y referencias sobre el equipo de investigación. Fue respondido de manera completa por 66 personas, luego de realizar algunas pruebas piloto para evaluar la calidad de la redacción y la efectividad comunicativa. Asimismo, se mantuvieron en el corpus las entrevistas realizadas, en virtud de su complementariedad.

3. Resultados. La provisión de cuidado no remunerado a personas mayores. Experiencias en primera persona.

\footnotetext{
"Tita sintió que una violenta agitación se posesionaba de su ser: enfrentó firmemente la mirada de su madre mientras acariciaba el chorizo y después, en lugar de obedecerla, tomó todos los chorizos que encontró y los partió en pedazos, gritando enloquecida.
}

1 En virtud del momento en que se realizó esta investigación, no se cuenta con un bagaje empírico apropiado para analizar las especificidades del cuidado no remunerado de personas mayores en pandemia, que ameritaría abordajes específicos. 
—iMire lo que hago con sus órdenes! ¡Ya me cansé! ¡Ya me cansé de obedecerla!"

Laura Esquivel, Como agua para chocolate, 1989

3.1. ¿Quiénes proveen cuidados no remunerados a personas mayores en Mar del Plata?

La disposición de las mujeres a proveer cuidados gratuitos se da por sentada. No alcanza a imaginarse lo que ocurriría si, como Tita, todas, al mismo tiempo, se hartaran de obedecer. Las políticas públicas de cuidado de personas mayores en Argentina tienen un corte fuertemente focalizado y familiarista, que refuerza la feminización. La provisión está concentrada en las situaciones de vulnerabilidad extrema y las personas mayores con carencia de redes de sostén familiar. Ello redunda en una sobrecarga de trabajo no remunerado hacia las mujeres.

El primer hallazgo de nuestra aproximación es que, entre las personas que respondieron al cuestionario, hubo un amplio predominio de mujeres, con un $92,4 \%$. Siguen a ello solo un $4,5 \%$ de varones; un caso de "no binarie" y uno que seleccionó la categoría "otros". Aunque la circulación del cuestionario tuvo una difusión amplia, podemos observar que los datos recabados en esta pregunta ponen en evidencia aquello que venimos desarrollando a lo largo de nuestra investigación: son las mujeres, casi siempre, las encargadas del cuidado de personas mayores. La predisposición mayoritariamente femenina para dar respuesta se vincularía así, con un proceso de autoidentificación o autoexclusión con la temática propuesta por el relevamiento. En cuanto a la edad, el rango fue amplio, entre los 21 y los 67 años, con un importante predominio de la franja etaria que va desde los 51 y 60 años. Ello expresa una sobrecarga preocupante que afecta a la salud de las mujeres (Battyány, Genta y Perrota, 2017; Larrañaga et al., 2008; Hernández Gómez et al., 2020) y una prolongación del tiempo vital dedicado a los cuidados ya que, saliendo de la edad reproductiva, se iniciaría un nuevo ciclo de cuidado destinado a proveer bienestar a la generación anterior. Asimismo, cabe advertir que se trata de mujeres en edad económicamente activa, lo que prolonga también la situación de doble jornada de trabajo (Hochshild, 1989; Wainerman, 2005). Ello intensifica y prolonga a lo largo de todo el ciclo vital 
la condición de pobreza de tiempo (Esquivel, 2009) en las mujeres y afecta la posibilidad de desarrollar proyectos de vida autónomos. En función del condicionamiento de las carreras laborales de las mujeres por el cuidado no remunerado, no es un dato menor ya que, en generaciones anteriores, el retorno al mercado de trabajo se asoció con la finalización del ciclo reproductivo (Wainerman, 2007). El aumento de la esperanza de vida, que en Argentina creció diez años entre 1970 y $2010^{2}$, reforzaría la demanda de este nuevo ciclo de cuidado.

En cuanto al nivel educativo alcanzado, los mecanismos de circulación digitales implican una sobre representación de los niveles educativos más altos, como se ha visto en otros estudios con metodologías similares (Crianza con Derechos, 2020; Viego y Actis, 2020): el 34,8\% de las personas entrevistadas alcanzaron nivel universitario/terciario incompleto, mientras que 31,8\% lo completó; el 10\% alcanzó nivel de posgrado; $19 \%$, secundario completo, solo $1,5 \%$, incompleto no lo completó; un solo caso de primario completo. Cabe advertir que, aún bajo estas condiciones de relativa ventaja, el cuidado de las personas mayores persiste como responsabilidad de las mujeres, con escasas oportunidades de redistribuir el cuidado no remunerado de manera más equitativa. Una amplia mayoría realiza estas tareas de cuidado bajo la modalidad de "doble jornada", ya que solo un 12,5\% expresó que se dedicaba de manera exclusiva al trabajo doméstico y de cuidado no remunerado. Para llegar a esta proporción se realizaron varias preguntas complementarias y se procuró un cruzamiento de los datos para contrarrestar el sesgo de autopercepción que tiende a sobre representar la condición de "ama de casa" cuando quienes responden son mujeres. La revisión de la estadística pública con perspectiva de género ha evidenciado la histórica tendencia a asociar la condición de mujer con la de ama de casa, tanto en las personas que responden, como en la construcción e interpretación de los datos (Wainerman y Rechini de Lattes, 1980; Cutuli, 2012). Este esfuerzo metodológico permite cuestionar la legitimación de una división sexual del trabajo 
parapetada en la presunta disponibilidad de tiempo de las mujeres, que se dedicarían al trabajo doméstico y de cuidado mientras los varones proveen los recursos necesarios para la subsistencia (Crompton, 1999). En suma, la asociación entre mujer y ama de casa habría subvalorado las actividades remuneradas de las mujeres en los registros estadísticos, lo que se reprodujo al observar algunos casos relevados en este estudio, donde se declaraba la condición de ama de casa y luego se incorporaban otras actividades.

\subsection{Caracterización de las personas mayores que reciben cuidados}

La siguiente sección de preguntas de la encuesta nos posibilitó adquirir información respecto a las personas mayores que reciben cuidados no remunerados. En ella se incluye el vínculo que la une con la cuidadora, la composición de la familia, las personas con las que vive, el estado de salud que presenta, la asistencia que requiere para el desarrollo de su vida diaria, entre otros. Los datos recabados en esta sección nos permitieron conocer el trabajo de cuidado que realizan las personas encuestadas y el tiempo destinado a él, como así también el nivel de dependencia de la persona mayor y la posibilidad de compartir el cuidado con otras personas.

Cuando consultamos respecto al vínculo que une a la persona mayor con la persona cuidadora, encontramos que el 51,5\% son personas que cuidan a sus madres; el 18,2\% realizan cuidados no remunerados a sus padres; el 16,7\% que cuida a sus abuelos/abuelas, y el resto a distintos familiares como tíos/as, hermanos/as, suegros/as, entre otros. A partir de estos resultados, se puede observar que es la familia quien se hace responsable de los cuidados de la persona mayor, desde el acompañamiento en la realización de trámites y compras, hasta la asistencia para la higiene personal o ingesta de alimentos.

En relación al estado de salud de las personas mayores, encontramos que, en una gran cantidad, muchos de ellos presentan una o más dificultades. El 68,2\% presenta los signos propios de la edad. El 39,4\% exhibe dificultades de movilidad, lo que implica una presencia aún más necesaria del cuidador. El 34,8\% posee enfermedades reumáticas tales como artritis, artrosis y osteoporosis. Continúan en prevalencia, 
un $25,8 \%$ de personas mayores con padecimientos de salud mental. Sin profundizar en cada tipo de trastorno, lo que ameritaría abordajes específicos, podemos inferir que existe una gran dificultad en la labor de las personas cuidadoras, ya que además de la carga física que los cuidados requieren, se suma el malestar emocional que implica asistir al deterioro de la salud de un ser querido. Por otro lado, el 22,7\% reconoce la existencia de enfermedades cardiovasculares; el 21,2\% disminución visual, el 16,7\%, disminución auditiva y el 12,1\%, enfermedades en el sistema respiratorio ${ }^{3}$.

Dentro de la muestra, podemos inferir diversos niveles de autonomía en las personas mayores, evidenciados a partir de la asistencia que las personas cuidadoras brindan para poder desarrollar las actividades de la vida cotidiana. En la mayoría de los casos, las personas mayores requieren asistencia en varios momentos de su vida diaria, a saber: para la preparación de alimentos el $27,27 \%$ precisa siempre de ayuda de la persona cuidadora; en la ingesta de alimentos solo el 7,57\% de los encuestados asiste a la persona mayor; el 18,18\% siempre requiere de asistencia para las tareas de higiene personal; el 15,15\% la necesita a la hora del control de esfínteres; el 31,81\% de las personas mayores precisa asistencia para la realización de trámites y compras; el 30,30\% recibe ayuda a la hora de administrarse su medicación; al igual que para los trámites, el $31,81 \%$ de las personas mayores necesita asistencia para los traslados fuera del hogar; mientras que el $22,72 \%$ la necesita para realizar chequeos médicos.

Como se puede observar, las personas encuestadas deben participar en una gran cantidad de tareas cotidianas de las personas mayores. De esta forma, si la persona responsable del cuidado no cuenta con relevo para distribuir las tareas, ya sea remunerado o no, podemos inferir que deberá resignar gran parte de su tiempo al desarrollo de estas actividades, relegando oportunidades laborales y tiempo libre.

En la pregunta sobre el lugar de residencia de las personas mayores, encontramos que un $37,9 \%$ vive con la persona cuidadora, mientras que el $62,1 \%$ no lo hace. Mediante diferentes mecanismos, ambas si-

3 Los porcentajes pueden sumar más de 100, ya que se trataba de una respuesta de opción múltiple. 
tuaciones imponen una disponibilidad de tiempo que limita la cotidianeidad de la persona cuidadora. Si hay convivencia, el tiempo se expande en virtud de la potencial demanda permanente de cuidado. Ante la no convivencia, los traslados se suman al tiempo dedicado al cuidado.

Respecto a la percepción de jubilaciones y pensiones, el 97\% de las personas mayores cuenta con algún ingreso. Sin embargo, el 43,9\% señala que no es suficiente para cubrir los gastos de la persona mayor. Ello redunda en situaciones en las que a la provisión de cuidado se suma la sobrecarga en términos de provisión económica, habitualmente sobre las espaldas de estas mismas personas cuidadoras. En lo que respecta a la atención sanitaria, en la totalidad de los casos relevados la persona mayor cuenta con obra social. El $68,18 \%$ cuenta con la cobertura de PAMI. Esta obra social estatal brinda servicios sociales a personas mayores desde 1971, proveyendo atención a partir de centros propios y prestadores públicos y privados, a cinco millones de personas, mayoritariamente jubilados y pensionados (PAMI, 2020). Le sigue en relevancia IOMA, una obra social estatal de la Provincia de Buenos Aires, que brinda cobertura a trabajadores y jubilados del sistema provincial (IOMA, 2020). Continúan, con escasa incidencia, algunas obras sociales sindicales que permiten la continuidad de las prestaciones a sus afiliados luego de la jubilación, y en menor medida, prestadores privados. Este es un dato relevante por varias razones. En primer lugar, las políticas de asistencia al cuidado -subsidios al cuidado domiciliario o en instituciones, que son escasos y mayoritariamente dirigidos a los casos donde se demuestra incapacidad de la familiase vehiculizan a través de las obras sociales. Veremos más adelante las oportunidades de acceso efectivo a esa protección. En segundo, la cobertura sanitaria opera como salario indirecto para las personas mayores $y$, teniendo en cuenta que, como veremos, las cuidadoras no remuneradas no solo proveen tiempo sino también recursos materiales para el sostén económico, el acceso a la obra social puede ser reflejo de una faceta donde la política pública tiene un nivel relevante de presencia, aunque las limitaciones de los servicios ameritarían estudios específicos entre los usuarios y sus familiares. 
3.3. ¿Cómo se organizan las tareas de cuidado no remunerado de personas mayores?

Si bien un gran porcentaje de personas mayores cuenta con una gran composición familiar que incluye a varias personas, las tareas de cuidado recaen en una sola de ellas, casi siempre mujer. Con relación a esto último, propusimos una serie de actividades cotidianas implicadas en el cuidado de personas mayores, para analizar las responsabilidades de cuidado con respecto a las actividades en las que éstas se comparten entre los distintos miembros de la familia o con algún personal remunerado. Dividimos las actividades en: preparación de alimentos, ingesta de alimentos, higiene personal, control de esfínteres, trámites y compras, administración de la medicación, traslados fuera del hogar y chequeo doméstico de salud, y consultamos sobre la persona encargada de cada una de ellas.

Entre las actividades que requerían asistencia, identificamos ciertos aspectos que consideramos oportuno analizar. Es evidente una generización de tareas aún en los casos en que hay varones involucrados. Las mujeres participan, en su mayoría, en la asistencia de las tareas relacionadas con la preparación de alimentos, la higiene personal, la realización de trámites y compras, la administración de la medicación y los chequeos domésticos de salud. Tareas que requieren mayor proximidad, disponibilidad afectiva y tiempo dedicado. En los casos en los que comparten la responsabilidad con otra persona, generalmente se trata de familiares mujeres o de personal remunerado; éstos últimos principalmente en las actividades de control de esfínteres e higiene personal. Por otro lado, podemos observar que las actividades en donde se distribuye más equitativamente la responsabilidad son las relacionadas con los trámites y compras y los traslados fuera del hogar, ya que se destaca un incremento en la participación de los varones en dichas tareas. Es decir, que hay algún grado de involucramiento masculino en las tareas con menor carga física y emocional y, además, desarrolladas en el espacio público. El cuidado asistencial y directo, que requiere cercanía física y contacto con el otro, con lo íntimo y escatológico, se confina a las mujeres. Como afirma Soledad Murillo (2006) en El mito de la vida privada, que indefectiblemente lo privado 
e incluso lo íntimo, para las mujeres constituyen esferas de trabajo, lo que refleja una enorme desigualdad con respecto a los varones y sus posibilidades de autonomía.

Luego de ello, indagamos sobre la dedicación a las tareas de cuidado. Es llamativo que la respuesta mayoritaria respecto a la justicia distributiva haya sido afirmativa, pese a identificar en el resto de las respuestas un notable nivel de sobrecarga de trabajo de cuidado no remunerado la mayoría considera que el trabajo del cuidado se distribuye de igual forma entre hombres y mujeres dentro de la familia. Ello sugiere una naturalización de los roles de género. Podemos intuir que las personas encuestadas se encuentran atravesadas por la idea que se sostiene y reproduce en la sociedad respecto a la identificación de los roles de género dentro y fuera del hogar. Persiste la asociación entre el trabajo doméstico y de cuidado y la feminidad, tal es así que a menudo se asume estas tareas como propias de las mujeres, y se tiende a justificar esta desigualdad bajo las condiciones biológicas de las mujeres, tal como mencionamos y desarrollamos en el capítulo uno. En relación a esto, observamos que existe un $69,7 \%$ de encuestados que desarrollan los cuidados de manera similar a las generaciones que los antecedieron sin que, como Tita, el personaje de nuestro epígrafe inicial, se atrevan siquiera a preguntarse sobre los fundamentos de tal tradición. Esta reproducción intergeneracional de las desigualdades favorece la continuidad de una "percepción de justicia" que se construye no tanto en virtud de las condiciones objetivas de la distribución de las responsabilidades como de la valoración sobre la situación ideal en términos de justicia distributiva (Thompson, 1991; Johnson y Blair, 1992; Greenstein, 1996). Asimismo, no podemos reducir la situación actual de la sobrecarga de trabajo de cuidado en las mujeres a una mera cuestión cultural. El marcado corte familiarista en el diseño de políticas constituye una variable crucial en su reproducción. El Estado -por acción u omisión- es un actor sustancial en el modo en que se despliega la organización social del cuidado.

Otro aspecto fundamental del sostén al cuidado es el acceso a la atención sanitaria de las personas mayores, como así también sobre el conocimiento que tienen las entrevistadas sobre los programas y prestaciones referidas al cuidado. En relación a lo primero, como men- 
cionamos antes, el $100 \%$ accede a cobertura, pero solo el $48,4 \%$ tiene conocimiento sobre programas o prestaciones que estén destinadas a garantizar o complementar el cuidado. En lo que respecta a las entrevistas presenciales, si bien tenían conocimiento, sin embargo, una de las entrevistadas advertía:

(...) averigüé en PAMI sobre unas cuidadoras que vinieran a domicilio un par de horas nomás (...) ya no las paga la obra social, daban un subsidio que en ese momento eran $\$ 800$ mensuales. (...) Luego, vinieron unas trabajadoras sociales de Buenos Aires a hacer un relevamiento en PAMI central, y yo tuve acceso porque me llamaron para una entrevista y me dijeron que yo tenía que solicitar un subsidio a la vejez. Y ese subsidio cuando lo fui a solicitar, era solo hasta $\$ 2500$, es decir, tenía que hacer una movida grande (...) llevarla a distintos médicos para que se vea el grado de dependencia que tiene mi mamá, pagar todas las ambulancias con lo que salía y demás, me pareció que no valía la pena por el monto del subsidio, porque además esto iba a ser evaluado luego, para ver si me lo podían otorgar o no (Camou, Colombo y Vinitzky, 2020).

El relato de Victoria expresa la realidad de muchas cuidadoras: no sólo existe más de un $50 \%$ de personas que no logra siquiera tomar conocimiento de los distintos programas o servicios que brindan las obras sociales, sino que el $48 \%$ que sí lo hace, encuentra dificultades a la hora de acceder a ellos y, cuando finalmente se accede, representan un monto insuficiente para paliar la sobrecarga del cuidado y precariza a las trabajadoras remuneradas que lo realizan. El acceso per se a la obra social no informa en profundidad las dificultades de efectivización de derechos, por lo requeriría abordajes específicos.

Por otra parte, no debe omitirse que un $40 \%$ de personas, en la pregunta sobre la justicia distributiva, cuestionaron este orden establecido. Es decir, que consideran que sí existe una desigualdad en la distribución de las tareas de cuidado y que éstas no se distribuyen equitativamente. Se atreven, como Tita, a advertir la injusticia que atraviesan, aunque ello no implica que dejen de padecerla. 
3.4. Impacto del cuidado no remunerado en las trayectorias vitales

Haremos referencia al impacto que el cuidado de personas mayores genera en la vida de las personas encargadas de él, es decir, cómo afecta esta carga en la vida laboral y en el bienestar personal de la persona responsable. Recuperando la convivencia, clave de la autonomía en el proyecto de vida propio, pudimos observar que el $66,6 \%$ de las personas encuestadas respondieron que no conviven con la persona mayor, sin embargo, el 33\% restante dio a conocer que la persona mayor viven en el mismo hogar. Asimismo, dentro del porcentaje de personas que no conviven con su cuidador, el 54,5\% vive solo. De estos casos, el $10 \%$ tres situaciones en particular donde la persona vive cerca de su cuidador, y en el $7 \%$ la persona mayor vive con cuidadoras remuneradas. El $15 \%$ de las personas que reciben cuidados no remunerados vive con su cónyuge y el $11 \%$ se encuentra institucionalizada en un hogar. También, se pudo observar que el $5 \%$ vive solo, pero en el mismo terreno que su cuidador. Un $9 \%$, convive con un hijo/a o nieto/a.

Consideramos pertinente resaltar estos porcentajes, porque si bien la mayoría de las personas encuestadas no convive con la persona mayor que cuidan, hay una gran parte que sí lo hace. Podemos inferir que la cuidadora decide convivir o vivir cerca de la persona mayor para facilitar su organización, teniendo en cuenta que la distancia termina siendo un condicionante, principalmente en casos de emergencia. Sin embargo, la contracara de esta cercanía es la intensificación del trabajo no remunerado de cuidado, generando que la cuidadora organice sus tiempos alrededor de las necesidades de la persona mayor.

En relación a esto último, la siguiente pregunta tenía como objetivo visualizar el tiempo que los entrevistados dedican al cuidado de la persona mayor. Las respuestas arrojaron que el 53\% de las entrevistadas le dedican todo el tiempo que se requiere y un $16 \%$ ya ha perdido la cuenta. Esta inconmensurabilidad del tiempo dedicado constituye un indicador crucial de cómo el cuidado atraviesa de manera transversal la vida de la persona que provee cuidados. Un 3\% respondió que cuida entre 6 y 9 horas por día, mientras que un 1,5\% señaló la opción de más de 5 horas por día. Los resultados obtenidos ponen en 
evidencia la inmensa e invisibilizada entrega de los cuidadores y cómo esto impacta en sus trayectorias vitales. Se relegan las propias carreras laborales y, aún más, las actividades de ocio y autocuidado.

El impacto en la vida de las personas cuidadoras, que genera sobrecarga y estrés, es consecuencia, en muchos casos, de no contar con un relevo en la responsabilidad de las tareas. Cuando preguntamos sobre ello, el $31,81 \%$ de los entrevistados nos respondieron que en pocas ocasiones solían contar con una persona que los reemplazara, mientras que el 13,63\% no cuenta con ningún tipo de relevo en el cuidado de la persona mayor.

En una de las entrevistas presenciales que realizamos antes de que comience el ASPO, Victoria nos dijo:

Por viajes no tengo relevo, y el resto nos arreglamos con mi marido. Si yo tengo que hacer doble turno en el trabajo o alguna otra actividad, él solo se encarga de atenderla en lo que es la alimentación, pero no de higienizarla, de cambiarla, etc. eso todo lo hago yo (Victoria, ciudad de Mar del Plata, 2020).

La ausencia de relevos en las tareas de cuidado constituye un factor desatendido por la política pública argentina, con una gran cantidad de cuidadoras no remuneradas de dedicación exclusiva. Ello afecta al despliegue de un proyecto autónomo así como a la calidad de vida y la salud de las personas que cuidan, con lo cual no es excesivo afirmar que se provee bienestar a la población dependiente a costa de la salud de las mujeres.

Por otro lado, indagamos sobre la situación económica de la persona mayor, refiriéndonos exclusivamente si estos contaban con pensión o jubilación con la que puedan costear sus necesidades, obteniendo que el $96 \%$ de los mismos si poseen. Sin embargo, más del $50 \%$ da cuenta de que estos haberes no son suficientes y que las personas mayores suelen recibir ayuda económica de su familia y principalmente, de su cuidadora principal. El objetivo de estas dos preguntas era visualizar que por más que la mayoría de las personas mayores cuenten con un ingreso, éste suele ser insuficiente, siendo nuevamente la familia la responsable de enfrentar dicha dificultad. Por ello, sumada a la 
sobrecarga de trabajo de cuidado no remunerado, la feminización de la pobreza se intensifica, en tanto las mujeres comparten sus escasos recursos con las personas que cuidan. Teniendo en cuenta la persistente brecha salarial (Actis de Pasquale y Lanari, 2015), ello constituye otro mecanismo de pauperización de las mujeres.

3.5. ¿Amor o trabajo? Representaciones en primera persona sobre el cuidado no remunerado

El objetivo del núcleo problemático es evidenciar la dificultad que se nos presenta a la hora de reconocer, cuestionar y transformar los roles que nos fueron asignados históricamente a las mujeres. El trabajo de cuidado de las mujeres dentro de los hogares nunca fue reconocido como tal, sino que se ocultó bajo las condiciones presuntamente propias de la esencia femenina, como son la afectividad y la asociación entre reproducción biológica y cuidado.

Para llevar adelante el desarrollo del presente apartado realizamos preguntas referidas a diferentes posibilidades de organización del cuidado. Con respecto a la idea de contratar un cuidador remunerado para alivianar la carga respecto a las tareas de cuidado, hay un $19,7 \%$ que cuenta con relevo remunerado. Asimismo, el 22,7\% pensó en hacerlo, sin embargo, el 12,1\% teme no poder solventar dicho gasto. Por otro lado, el $12,1 \%$ de las personas cuidadoras que desearía contar con relevo, respondió que no lo hace ya que la persona mayor no está de acuerdo.

Esto último nos llevó a reflexionar acerca de lo arraigada que se encuentra la idea de la familia como principal o único cuidador de la persona mayor, así como la posible asociación entre la contratación de personal externo con el reconocimiento de la pérdida de autonomía. La asociación entre familia y cuidado constituye un freno simbólico para la distribución del cuidado, profundizado por limitantes materiales. Con ello, no es de extrañar que el 30,3\% no haya pensado siquiera en la posibilidad de contar con cuidado remunerado ya que, como mencionamos anteriormente, el $70 \%$ organiza las tareas de cuidado de manera similar a las generaciones anteriores. Por último, creemos central destacar que el $10,6 \%$ se considera como la única responsable 
de dichas tareas. En la siguiente pregunta obtuvimos resultados similares, ya que el $47 \%$ de las personas reconoce que no pensó siquiera en la idea de institucionalizar a la persona mayor. Por otro lado, el $22,7 \%$ considera que ésta debe estar en su propio hogar, mientras que el 10,6\% reconoce haberlo pensado, pero es la persona mayor la que no está de acuerdo.

Estas respuestas nos permiten pensar, nuevamente, en la deficiente distribución de la organización social de los cuidados, que delega la responsabilidad de forma casi exclusiva a las familias, desligando al resto de los actores de dicha actividad. A su vez, el cuidado de personas mayores es invisibilizado por quienes no lo realizan, estableciendo "la entrega personal de las mujeres a una actividad sin valor en la medida en que no se rige por las normas de la producción regulada por el mercado" (Sánchez Reinón, 2012:201).

Para concluir, el 78,8\% de las personas encuestadas considera que el trabajo de cuidado de la persona mayor no debe ser remunerado, viéndolo como una tarea que corresponde hacer de manera gratuita, argumentando entre otras cosas que:

Es una tarea que realizo desde la convicción y con mucho amor.

Es mi papá y lo hago por amor.

Es mi mamá, ¿por qué cobrar?

Nunca le cobraría a mi madre, hago lo que ella hizo por mi cuando era chica.

Es una forma de retribuirle.

No, porque es mi madre y debo hacerlo.

No, porque corresponde por ser mi madre (Personas encuestadas, ciudad de Mar del Plata, 2020)

Estas afirmaciones acerca de qué fundamenta el cuidado no remunerado transita tres lógicas vinculares: amor, reciprocidad y deber. Por diversos mecanismos simbólicos, las tres garantizan la legitimidad de la provisión gratuita de cuidados o, en otros términos, la extracción sin pago de la fuerza de trabajo de las mujeres. Viviana Zelizer (2009) advierte las implicancias de género de la construcción de una moral del dinero que impone de modo ficticio una demarcación entre los 
vínculos monetarios y afectivos como mutuamente excluyentes. La tensión entre amor y trabajo, reforzada desde el feminismo en aras del reconocimiento del valor del cuidado (Federici, 2013) obliga a las mujeres a elegir una u otra pues, esta moral del dinero expresaría -solo para ellas- la ausencia de amor cuando se exige retribución y, como contraparte, la exclusión de la retribución afectiva cuando media la retribución económica. En términos de reciprocidad, cabe advertir la generización de los modos de integración económica ${ }^{4}$ (Polanyi, 2013), que subyace a este mandato. Una relectura en clave de género de $\mathrm{La}$ gran transformación, nos sugiere que el imperio del mercado no ha eliminado otros modos de integración económica. Las desigualdades que provoca el "molino satánico" se agudizan si las pensamos en clave de género, ya que el proceso de mercantilización se ha parapetado en la persistencia de relaciones sociales excluidas de las injusticias del mercado, pero también de sus beneficios. Así, la reciprocidad necesaria para sostener los cuidados en los extremos de la vida es, en efecto, asimétrica, puesto que todas las personas los reciben, pero solo las mujeres los proveen. Cabe advertir asimismo la familiarización implícita al señalar que "nunca le cobraría a mi madre", asumiendo el cuidado como una relación social de la esfera familiar y en la que nadie más está involucrado, ni los otros familiares, ni la comunidad, ni el Estado. Finalmente, en versión más cruda de la lógica patriarcal, algunas declaran que "corresponde" y "debo hacerlo". Cumplen, más allá de sus propios deseos y necesidades, con aquello que se espera de ellas. Constituye un deber moral, mas no es universal sino femenino (Guilligan, 1982; Benhabib, 1992). El campo de estudios sobre la socialización generizada ha profundizado lo suficiente en ello (Nari, 2005). Este esfuerzo por reproducir intergeneracionalmente la provisión gratuita de cuidados conforma un esfuerzo tan temprano e intenso en la vida de las mujeres, que lleva toda una vida cuestionarlo, si es que se llega a hacerlo. 
Asimismo, es de destacar que el mandato de gratuidad admite fisuras y, como la protagonista de nuestro epígrafe, el 18,1\% pone en cuestión la hegemonía de este mandato. Así, declaran que el cuidado intrafamiliar de personas mayores sí debería ser remunerado, manifestando que:

Sí, porque es un trabajo desgastante. Sí, porque requiere tiempo y formación. Sí, porque siendo la única cuidadora, tuve que renunciar a mi trabajo para poder cumplir con ese rol. Deberían ser remuneradas porque sería más equilibrado. Son tareas invisibles que recaen principalmente en nosotras, limitando nuestro desarrollo personal y económico, y disminuyen nuestra calidad de vida y roban tiempo y energía para dar amor al familiar del que nos hacemos cargo, ya que la relación se transforma en una rutina despersonalizada (Camou, Colombo y Vinitzky, 2020a).

Desarticulando sentidos comunes, debería ser absurdo preguntar las razones por las que se debería retribuir un trabajo. En palabras de Reva Siegel: "vivimos en un mundo en el que el trabajo no remunerado en el hogar permanece con una anómala falta de justificación y ni siquiera parece requerir una" (1994:210). Aquí, en primera persona, las cuidadoras no remuneradas exponen por qué su tarea debería tener un reconocimiento económico. Se ensayan fundamentos en cuatro líneas: el carácter de trabajo de la actividad; el coste de oportunidad de su realización; la justicia social/de género y, por último, una sugerente subversión entre amor y trabajo. En cuanto al carácter de trabajo del cuidado, se refiere al tiempo y al esfuerzo, demandas reconocimiento de larga data de la economía feminista (Rodríguez y Cooper, 2005; Rodríguez Enríquez, 2015), mas también al conocimiento necesario para desempeñarlo. De esta manera, se pone en tela de juicio el discurso en que se parapeta la generización de las tareas de cuidado, construyéndolas simbólicamente como descalificadas e intrínsecas a la condición femenina. En segundo término, y en parte como continuidad del razonamiento anterior, se recupera la valoración en función del coste de oportunidad. Si el cuidado es un trabajo que demanda tiempo, esfuerzo y saberes, desplegarlos en él impide hacerlo en otros 
espacios. Es decir, el valor se torna indirecto, en virtud de la carencia que provoca. Ello no es un dato menor para el sesgo educativo que conforma esta muestra, en la medida que a mayores niveles de calificación formal, el costo de oportunidad de resignar participación en el mercado laboral es mayor. En cuanto a la justicia de género, se advierte que de la mano del reconocimiento del valor del cuidado viene la demanda de la redistribución (Fraser, 1996). Finalmente, se destaca una profunda resignificación del discurso que legitima la gratuidad. Si en nombre del amor la gratuidad se vuelve mandato, cabe advertir si la propia obligación no socava el lazo afectivo. La abnegación es no solo un problema para las mujeres proveedoras de cuidados (Friedan, 2009), sino también para quienes los reciben, y para los lazos afectivos que se tejen entre ellos.

\title{
Conclusiones
}

\begin{abstract}
"Sólo Tita, cuya misión era la de atenderla hasta su muerte, era la única que podía estar presente en el ritual y ver a su madre desnuda. Nadie más. Por eso se había construido este cuarto a prueba de mirones. Tita le tenía que lavar a su mamá primero el cuerpo, luego el cabello y por último la dejaba unos momentos descansando, gozando del agua, mientras ella planchaba la ropa que se pondría Mamá Elena al salir de la tina.

A una orden de su madre, Tita le ayudaba a secarse y a ponerse lo más pronto posible la ropa bien caliente, para evitar un resfrío. Después, entreabría un milímetro la puerta, para que el cuarto se fuera enfriando y el cuerpo de Mamá Elena no sufriera un cambio brusco de temperatura. Mientras tanto le cepillaba el pelo, alumbrada únicamente por el débil rayo de la luz que se filtraba por la rendija de la puerta y que creaba un ambiente de sortilegio al revelar las formas caprichosas del vapor de agua. Le cepillaba el cabello hasta que éste quedaba seco por completo, entonces le hacía una trenza y daban por terminada la liturgia."
\end{abstract}

Laura Esquivel, Como agua para chocolate, 1989

Como los que realizaba Tita para Mamá Elena, los cuidados son repetitivos, agotadores, requieren intimidad y proximidad, y son imprescindibles para la sostenibilidad de la vida. Realizarlos en exclusiva priva de un proyecto de vida propio y autónomo. La actual organización social de los cuidados impone un dilema donde el abandono 
está siempre presente. Si el Estado puede reconocer ausencia -abandono- familiar, se hace presente con suficiente intensidad. Si, como en los casos aquí analizados, las familias -léase "las mujeres"- recogen el guante del deber de cuidar, el Estado las abandona y se convierten en cuidadoras de responsabilidad casi absoluta.

A lo largo de este artículo se visibiliza la complejidad del cuidado, la cantidad de tiempo y esfuerzo que insume, así como la sobrecarga de estas tareas en las mujeres, y las limitaciones vitales que ello les impone. La familiarización del cuidado, y más aún más, en el cuidado de personas mayores, se cimienta en su feminización. Si, durante décadas, el discurso legitimador giró en torno a las cualidades naturales, a la mayor predisposición de tiempo por su condición de amas de casa, a la reproducción de tradiciones familiares, pareciera que el cuestionamiento de ninguna de esas realidades alcanza para revertir la feminización de los cuidados. Ya sea en forma de relegación del trabajo remunerado o de doble jornada laboral, la revolución sigue estancada. Y su impacto no es mensurable únicamente con la vara del mercado de trabajo, ya que además de oportunidades laborales, las mujeres que son absorbidas de lleno por el cuidado pierden experiencias vitales para el despliegue de proyectos de vida autónomos -actividades recreativas y formativas, viajes, vida social- y se ven expuestas a un desgaste físico y emocional que atenta contra su salud.

Así, un sistema social que descarga todo el volumen de cuidado en un único sujeto social es intrínsecamente injusto. $Y$ en tal caso, no se trata tanto de evitar la dedicación exclusiva al cuidado, aunque también, sino, sobre todo, poner en cuestión su inevitabilidad. Ésta queda plasmada en las lecturas legitimadoras de las mujeres que ejercen esa función, pero más aún de las que se atreven a cuestionar este orden social. Porque, en definitiva, poner en cuestión una organización social del cuidado injusta implica convivir con una disonancia. Acaso por eso Tita sufría como sus antepasadas no lo habían hecho. Sin embargo, esos cuestionamientos todavía minoritarios, constituyen el germen de la construcción de una demanda política que se traduzca en una diversificación de los mecanismos mediante los que se provee el cuidado.

$¿$ Amor o trabajo? Las demandas del feminismo necesitan soslayar la trampa en que se parapeta la subalternización de las mujeres. Como 
señala una de las entrevistadas, el trabajo incondicional, sin reconocimiento ni relevo, atenta contra el amor. La justicia social y de género, mas también la sostenibilidad afectiva de nuestra sociedad, dependen de la construcción de estructuras más equitativas de cuidado. Si la necesidad de cuidado es intrínsecamente universal, los mecanismos que lo proveen también deben serlo.

\section{Referencias bibliográficas}

Actis Di Pasquale, Eugenio y Lanari, María Estela (2015). El mercado laboral escenario de desigualdades persistentes. Las brechas abiertas de Argentina. Lanari, María Estela y Hasanbegovic, Claudia, (Eds.), Mujeres de Latinoamérica. El presente en veintidós letras (213-270). Mar del Plata, Argentina, EUDEM.

Batthyány, Karina; Genta, Natalia, y Perrotta, Valentina (2017). El aporte de las familias y las mujeres a los cuidados no remunerados en salud en Uruguay. Revista Estudios Feministas, 25(1), 187-213. Recuperado de: https://www.scielo.br/scielo. php?pid=S0104-026X2017000100187\&script $=$ sci arttext\&tlng=es

Benhabib, Seyla (1992). Una revisión del debate sobre las mujeres y la teoría moral. Isegoría, (6), 37-63. https://doi.org/10.3989/isegoria.1992.i6.323

Collins, Patricia (2000) Black Feminist Thought. Nueva York, Estados Unidos, Routledge.

Crianza con Derechos (2020). Encuesta realizada a personas responsables del cuidado de niños/as en edad escolar. Secretaría de Extensión, Facultad de Ciencias de la Salud y Trabajo Social, Universidad Nacional de Mar del Plata: Mar del Plata. Recuperado de: https://drive.google.com/file/d/1tnxENTGFsxyNNMIEZYhn i59 hgtiDgi/view? fbclid=IwAR27LCKJ6-WAYnFZovqmfEUV 54kuVwjPlA4TiemvgACNLJdSc3522B4snM

Crompton, Rosmarie (1999). Restructuring gender relations and empleyment: the decline of the Male Breadwinner. Oxford, Reino Unido, Oxford University Press.

Cutuli, Romina (2012). Medir es conocer. Economía feminista y cuantificación del trabajo. Observatorio Laboral Revista Venezolana, 5(9). Recuperado de: http:// servicio.bc.uc.edu.ve/faces/revista/lainet/lainetv5n9/art01.pdf

Esquivel, Valeria (2009). Usos del tiempo en la ciudad de Buenos Aires. Buenos Aires, Argentina, UNGS.

Greenstein, Theodore (1996). Gender ideology and percepcion of the fairness of the division of household labor: effects on marital quality. Social Forces, 74(3). 10291042. DOI: https://doi.org/10.2307/2580391

Grushka, Carlos (2014). Casi un siglo y medio de mortalidad en la Argentina. Revista Latinoamericana de Población, 8(15), 93-118. Recuperado de: https://dialnet. unirioja.es/servlet/articulo? codigo $=5349624$

Guilligan, Carole (1982). In a different voice. Psychological theory and women's development. Harvard, Estados Unidos, Cambridge. 
Hernández Gómez, Mercedes; Domínguez, María José Fernandez; Blanco Ramos, Manuel; Alves Pérez, María Teresa; Fernández Domínguez, María Jesús; Souto Ramos, Ana; González Iglesias, María Pilar y Fontán, Ana Clavería (2020). Depresión y sobrecarga en el cuidado de personas mayores. Revista Española de Salud Pública, 93. Recuperado de: http://scielo.isciii.es/scielo.php?script=sci arttext\&pi$\mathrm{d}=\mathrm{S} 1135-57272019000100089$

Hernández Sampieri, Roberto (2014) Metodología de la investigación. México, Mc Graw Hill Education.

Hochshild, Arlie (1989) The second shift. New York, Estados Unidos, Viking.

INDEC (2010). Censo 2010. Recuperado de: https://www.indec.gob.ar/indec/web/ Nivel4-Tema-2-41-135

INDEC (2014). Población por sexo y grupos quinquenales de edad. Años 20102040. Recuperado de: https://www.indec.gob.ar/indec/web/Nivel4-Tema-2-24-84

Johnson, Michael y Blair, Sampson (1992). Wives' perception of fairness and the division of household labor. Journal of Marriage and the Family, (54), 570-581. DOI: https://doi.org/10.2307/353243

Larrañaga, Isabel; Martín, Unai; Bacigalupe, Amaia; Begiristáin, José María; Valderrama, María José y Arregi, Begoña (2008). Impacto del cuidado informal en la salud y la calidad de vida de las personas cuidadoras: análisis de las desigualdades de género. Gaceta sanitaria, 22(5), 443-450.

Masanet, Erica y La Parra, Daniel (2011). Relación entre el número de horas de cuidado informal y el estado de salud mental de las personas cuidadoras. Revista Española de Salud Pública, 85(3), 257-266.

Murillo, Soledad (2006). El mito de la vida privada. De la entrega al tiempo propio. Madrid, España, Siglo XXI.

Sanchez Reinón, Manuel (2012). Los cuidados informales de larga duración en el marco de la construcción ideológica, societal y de género de los Servicios Sociales de Cuidado. Cuaderno de relaciones laborales, 30(1), 185-210. Recuperado de: https:// revistas.ucm.es/index.php/CRLA/article/view/39120

Siegel, Reva (1994) The modernization of marital status law: adjudicating wives. Rigths to earning, 1860-1930. Goergtown law journal, (82), 2127-2212. Recuperado de: https://www.law.yale.edu/sites/default/files/documents/pdf/Faculty/Siegel TheModernizationOfMaricalStatus.pdf

Thompson, Linda (1991). Family work. Women's sens of Fairness. Journal of Family, 12 (2), 181-196. DOI: https://doi.org/10.1177/019251391012002003

UN. Naciones Unidas (2021). Envejecimiento. Recuperado de: https://www.un.org/ es/sections/issues-depth/ageing/index.html

Naciones Unidas (2015). World Population Ageing 2015, Department of Economic and Social Affairs, Population Division. New York: United Nations. Recuperado de: https://www.un.org/en/development/desa/population/publications/pdf/ageing/ WPA2015 Report.pdf

Viego, Valentina y Actis Di Pasquale, Eugenio, (Eds.) (2020). Encuesta iberoamericana sobre Rutinas Laborales y Cotidianas en Tiempos de COVID-19. Principales resultados. Cádiz, TRAGEVIC. Recuperado de: http://nulan.mdp.edu.ar/3442/

Wainerman, Catalina (2005) La vida cotidiana en las nuevas familias, ¿una revolución estancada? Buenos Aires, Argentina, Lummiére. 
Wainerman, Catalina (2007) "Mujeres que trabajan. Hechos e ideas". En S. Torrado (Ed.) Población y bienestar en Argentina del primero al segundo centenario (325-252). Buenos Aires, Argentina, Edhasa.

Wainerman, Catalina, y Rechini de Lattes, Zulma (1980). La medición del trabajo femenino. Buenos Aires, Argentina, CENEP.

Recibido: 18/07/2021

Aceptado: 05/10/2021 